\title{
¡LO FUE! LA COMPETITIVIDAD INTERNACIONAL DE LA SIDERURGIA VIZCAÍNA. CONTESTACIÓN A ANTONIO ESCUDERO
}

\author{
STEFAN HOUPT \\ Universidad Carlos III de Madrid ${ }^{\mathrm{a}}$
}

Debo admitir que me ha costado reconocer mi hipótesis de trabajo, su contraste y mis conclusiones tal y como aparecen reflejadas en la nota que publica Antonio Escudero en este número. Trataré en pocas líneas de centrar el debate de nuevo en el tema de mi artículo y explicar algunos de los aspectos que pueden haber llevado a una lectura confusa de mi investigación.

En primer lugar, enunciaré de nuevo mi hipótesis de trabajo, la gran pregunta que sirve de hilo conductor a la investigación presentada. Mi hipótesis es que la industria siderúrgica española moderna pudo elegir producir para dos mercados, el nacional y el internacional, lo que podía haber generado una industria más competitiva con economías de escala, precios de acero más bajos y una pieza menos en la posterior nacionalización de los mercados españoles.

El contraste de la hipótesis se llevó a cabo comparando costes de producción de las fábricas españolas más competitivas con los precios de venta en los mercados de sus mayores competidores internacionales. Este ejercicio se realizó para

a Departamento de Historia Económica e Instituciones; Calle Madrid, 126; 28903 Getafe; España. Correo electrónico: shoupt@clio.uc3m.es 
identificar si existían productos competitivos, determinar sus características y su margen de competitividad. En definitiva, lo que se trató de comprobar era si las fábricas españolas podrían haber participado en los mercados internacionales.

Los datos internacionales que empleé para el contraste proceden de los trabajos de Allen (1975), Burnham y Hoskins (1943) y McCloskey (1973) sobre comparaciones entre las industrias de Estados Unidos, Alemania, Gran Bretaña y Bélgica. Los datos para España proceden de la contabilidad de costes de las fábricas en cuestión -las fábricas de Sestao y Baracaldo de Altos Hornos de Vizcaya. Son datos primarios, datos mensuales muy detallados para 59 productos y un periodo de 30 años $^{1}$. Estos datos se contrastaron con otras referencias a costes que se contienen en la documentación reservada de las empresas -Actas del Consejo de Administración, Actas de la Comisión Delegada del Consejo de Administración y Actas de la Comisión de Madrid.

No existe nada que nos pueda inducir a creer que los sucesivos equipos directivos de las empresas falsificaran de forma sistemática y mes a mes estos datos situándolos por debajo de los reales. Es decir, debemos partir del supuesto de que éstos son los datos que reflejan los costes de las empresas en cuestión. Partiendo de esta base, los nuevos cálculos del Profesor Escudero se reducen a una comprobación de mi contraste con datos recogidos de fuentes secundarias. Desgraciadamente, las discrepancias entre los datos primarios y secundarios no se analizan en la nota presentada, aunque sí puedo lanzar la conjetura de que los datos que recogieron los ingenieros franceses son equivocados y que fueron engañados sistemáticamente para ocultar el potencial de las fábricas en cuestión y guardar en secreto el inmenso poder de producir muy por debajo de todos sus competidores nacionales e internacionales en caso de tener que defender sus mercados cautivos y altamente lucrativos. Esto es todo lo que tengo que decir en relación a una posible revisión de mi trabajo publicado en esta Revista (Houpt, 2003, pp. 335-371).

Dentro del espíritu del avance de la ciencia y de las reglas de debate académico, añadiré unas líneas acerca de las valiosas observaciones que hace mi colega Antonio Escudero y unas aclaraciones menores que pueden contribuir a encauzar el futuro debate del tema.

Mi artículo no discute las cifras de exportación y producción de lingote, que ya es un debate cerrado con las actuales fuentes de datos y estéril y exhausto ad extremum. Se incluyeron datos de exportación de hierro y acero para mostrar que

\footnotetext{
${ }^{1}$ La fuente de los datos utilizados para los contrastes está indicada debajo del Gráfico 1 de mi artículo: se trata de los datos deflactados que contiene mi tesis doctoral (Houpt 1998). Miguel Ángel Saiz García, también profesor de la Universidad de Alicante, ha tenido la ocasión de consultar no solamente el primer volumen de mi tesis, que contiene una descripción pormenorizada del contraste, sino también las 568 páginas del segundo volumen, precisamente las que presentan estos datos primarios deflactados y que podrá confirmarle al Profesor Escudero el detalle, el rigor y la regularidad de estas series.
} 
no contradicen en ningún momento la hipótesis de que se pudieron haber exportado ciertos productos de hierro y acero, de ahí mi caveat -inadvertido- de hacer solamente un uso indicativo de los datos, que sirvan para dar una idea de la evolución de la salida de productos.

Alzola no indica en la cita proporcionada que los productos de mayor valor añadido habían de venderse en el mercado nacional. Pero sí es un gran connaisseur de las fábricas bilbaínas, como muestra su memoria, y quizás sus cifras no anden demasiado alejadas de la verdad ${ }^{2}$. Resulta difícil creer que un ingeniero como él se invente cifras conociendo las reales. Además, como fiel seguidor de Friedrich List, defendería la pauta alemana de proteger y producir para el mercado nacional aquello para lo que no tenían ventaja comparativa con el fin de alcanzar de esta forma la integración vertical y las economías de escala necesarias para desarrollar y competir en los mercados internacionales con los productos en los que sí la tenían ${ }^{3}$.

El lingote de Cleveland \#3 -el lingote «más barato del mundo»-es un lingote de fundición, no pudo ser empleado para fabricar acero de forma económica, ni siquiera acero Siemens-Martin básico o Thomas básico, ya que contenía demasiado sílice y azufre y la cantidad incorrecta de fósforo. Cuando se resolvieron estos problemas técnicos, los minerales de Cleveland se estaban agotando ${ }^{4}$. El lingote de Cleveland no se presta para las comparaciones que presenta el Profesor Escudero. Yo lo había incluido entre los precios internacionales del lingote como una referencia de precio mínimo y para sesgar el contraste en contra de mi hipótesis de trabajo.

La generalización sobre el triunfo de los sistemas básicas en Europa frente a los sistemas ácidos en declive expuesta en la nota es herencia de Burn (1940, pp. 16782) para explicar el declive de la industria de acero de Gran Bretaña; fue repetida por Burnham y Hoskins, Landes, Aldcroft y reexaminada y cuestionada desde los años setenta por McCloskey, Allen, Tolliday y Elbaum. Todos los revisionistas de esta vieja teoría coinciden en que no fue la adhesión de Gran Bretaña al sistema Bessemer ni siquiera a los sistemas ácidos lo que provocó su pérdida de competitividad y declive. La teoría que recoge hoy el estado de la cuestión es la de una excesiva fragmentación estructural de la industria, que impidió la necesaria integración

\footnotetext{
${ }^{2}$ Alzola fue accionista de cierta importancia de Altos Hornos de Bilbao y consejero desde 1894, representó a la Sociedad en la fusión con La Vizcaya y La Iberia en 1901-2, y fue nombrado Consejero Vicepresidente de la Comisión Delegada de Altos Hornos de Vizcaya a partir de entonces. Publicó en 1909 una de las monografías más detalladas que existen sobre las dos factorías.

${ }^{3}$ Webb (1980). En todo caso, en 1904 Alzola identificó los fuertes recargos sobre el carbón y los impuestos como los factores que impedían la exportación de productos siderúrgicos españoles. Véase Alzola (1904, p.40).

${ }^{4}$ Veáse Tolliday (1991), pp. 37-43 o Wengenroth (1986), pp. 37 n34, 190-91. La reducción de sílice, necesario para no dañar el revestimiento ácido, aumenta la presencia de azufre en el lingote, cuya eliminación es muy costosa.
} 
vertical y la creación de economías de escala ${ }^{5}$. Esta teoría es la que también defiendo para el caso de España: competir en los mercados internacionales podría haber proporcionado la demanda y las economías de escala necesarias para obtener un acero más barato con todo lo que podía haber conllevado para la economía española. Competir con aceros Bessemer contra los nuevos aceros era factible en las líneas de producto defendidas, y más todavía con las excelentes hematites de Vizcaya. Otra cuestión bien distinta es el agotamiento de las minas cuyos efectos deben ser analizados aparte.

Por último, y con ánimo de adelantar solamente una pequeña parte de mis investigaciones en curso y reservar sus resultados para futuras publicaciones, quiero presentar lo que el Profesor Escudero considera la prueba de carga sobre la competitividad del lingote y los raíles vizcaínos: el contraste entre costes en Bilbao y los costes en las fábricas extranjeras, pero realizado con datos primarios y homogéneos.

Los datos empleados para este contraste proceden de la documentación primaria y reservada de las empresas siderúrgicas españolas -Actas del Consejo de Administración, Actas de la Comisión Delegada del Consejo de Administración, Actas de la Comisión de Madrid y Contabilidad de Costes- publicados en mi tesis doctoral (Houpt 1998), y de los datos recogidos en los archivos históricos que albergan los libros correspondientes de las empresas extranjeras mencionadas, Historisches Archiv Krupp (Essen) y Glamorgan Record Office (Cardiff).

\section{CUADRO 1}

\section{COSTE DE PRODUCCIÓN DE LINGOTE BESSEMER EN LA FÁBRICA DE BARACALDO DE ALTOS HORNOS DE VIZCAYA) Y LAS FÁBRICAS DE CARDIFF Y DOWLAIS DE LA DOWLAIS IRON COMPANY} (Pesetas)

\begin{tabular}{|c|c|c|c|}
\hline Año & Baracaldo & Cardiff & Dowlais \\
\hline 1900 & 84,1 & 99,1 & \\
\hline 1904 & 69,7 & 81,7 & 79,42 \\
\hline 1908 & 61,54 & 78,1 & 80,29 \\
\hline
\end{tabular}

Fuente: Contabilidad de Costes Altos Hornos de Vizcaya en Houpt (1998), Vol. II y Glamorgan Record Office D/DG/C4 11, 12-19.

Nota: El dato de 1900 es el precio más alto para Baracaldo en el intervalo 1897-1916.

${ }^{5}$ Elbaum (1986, pp. 53-4). Difícilmente se explican las exportaciones vizcaínas de mineral de hierro de las primeras décadas del siglo XX a Gran Bretaña, si no era posible hacer acero a un precio competitivo con ellas. Recordemos que Gran Bretaña era una economía abierta, expuesta a la competencia externa de bienes producidos de forma más económica. El cincuenta y nueve por ciento del tonelaje de la navegación mundial en 1913 se construía en Gran Bretaña y la mayor parte con planchas, estructuras y motores de acero ácido. 


\section{GRÁFICO 1}

PRECIO DE COSTE DEL ACERO BESSEMER (Pesetas)

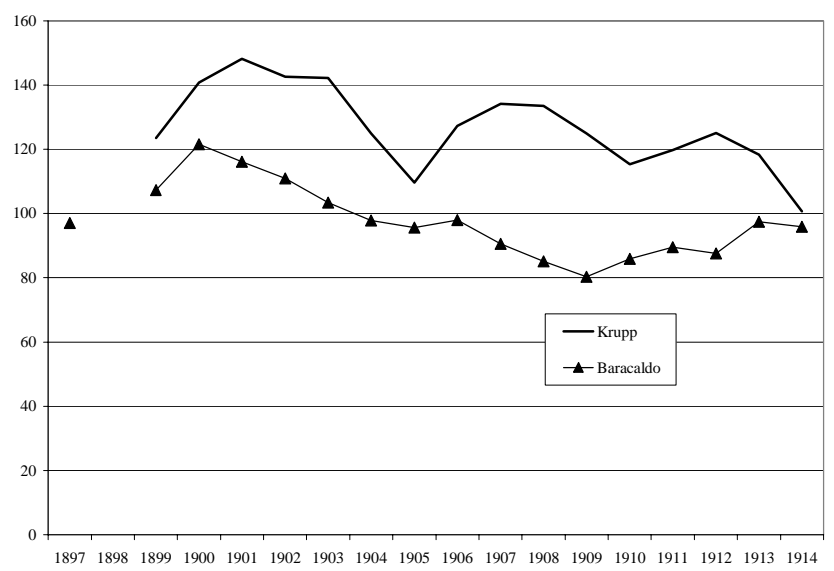

Fuente: Houpt (1998, Vol. I, pp.84-89), HA Krupp, WA 1805 \& 1609.

GRÁFICO 2

\section{NÚMERO DE CARGAS REALIZADAS EN LOS CONVERTIDORES BESSEMER}

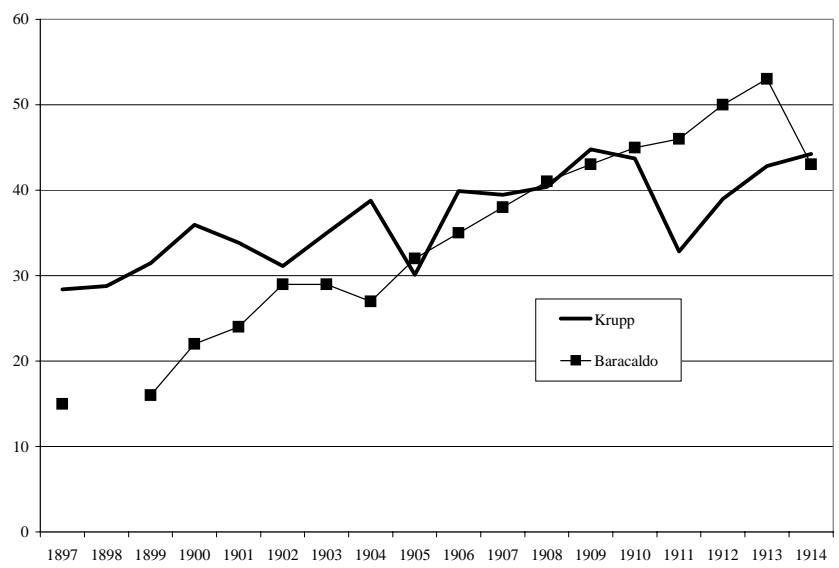

Fuentes: Houpt (1998, Vol. I, pp.84-89), HA Krupp, WA 1805 \& 1609. 
La comparación de precios de coste de lingote de hierro entre las fábricas de Baracaldo, Cardiff y Dowlais revela importantes diferencias. Si tenemos en cuenta que procesan el mismo mineral (Orconera) y que el consumo de carbón, tanto para la reducción del hierro como para la inyección de aire, es más caro en Bilbao, el resultado es realmente sorprendente. Baracaldo produce a un coste muy inferior. La obsolescencia de los equipos galeses no es la clave de esta historia dado que los hornos altos de Cardiff se construyeron entre 1891 y 1897 y los de Dowlais se modernizaron en 1904. Así pues, los hornos altos de Baracaldo superan a su homólogos de forma muy notoria.

El Gráfico 1 nos muestra cómo los precios de coste del acero Bessemer se sitúan de forma sistemática por debajo de los de Krupp, aun antes de alcanzar la eficiencia del uso del equipo que mantenía Krupp en 1906. El acero Bessemer de Baracaldo era muy competitivo y su siguiente transformación, los raíles, deberían recoger esta ventaja al partir de una materia prima (acero Bessemer) muy económica.

El Gráfico 2 muestra cómo el número de cargas realizadas medidas en unidades de convertidores equivalentes de la fábrica de Essen de Krupp y Baracaldo de AHV están a la par entre 1906 y 1915. Esto es más significativo aun si tenemos en cuenta que, aparte de emplear los mismo equipos suministrados por la empresa Galloway, procesan un lingote de hierro adecuado para su tratamiento mediante el procedimiento Bessemer obtenido con el mismo mineral Orconera, es decir, que son procesos totalmente homogéneos. El gráfico nos muestra que la fábrica de Baracaldo está a la altura de Krupp en la técnica de aprovechar al máximo la intensidad de uso de los equipos, y sus precios de coste reflejan que obtiene una eficiencia incluso superior a la de su homólogo alemán, una de las empresas líder de la siderurgia europea.

Dado el coste tan ventajoso al que produce acero Bessemer, la materia prima clave para producir raíles, me atrevo a sostener también mi hipótesis acerca de la competitividad de los raíles vizcaínos, incluso hasta la altura de la Segunda Guerra Mundial. Debo concluir, como hizo Alzola en 1905, que «aún se exportan carriles, a pesar de hallarse muy gravada la salida contra todos los principios económicos» ${ }^{6}$.

No puedo terminar aquí sino de la misma forma que en mi artículo. Dos empresas extranjeras con instalaciones similares a las de Altos Hornos de Vizcaya, que empleaban el mismo mineral de hierro, supieron aprovechar los mercados internacionales. La empresa alemana Krupp, una de las empresas líderes de la siderurgia alemana, superó la crisis económica iniciada en 1873 con ventas al extranjero, estrategia que supo concentrar en la exportación de raíles a los Estados Unidos de América en los años noventa. Exportó el 64 por 100 de su producción total entre 1879 y 1889 y el 33 por 100 entre 1890-19117 . Dowlais, una de las siderúrgi-

\footnotetext{
${ }^{6}$ Alzola (1905), pp. 22-3.

${ }^{7}$ Krupp (1912), pp. 223-4.
} 
cas más importantes de Gran Bretaña, llevó a cabo fuertes inversiones en una planta nueva y en la modernización de sus talleres de laminación para mantenerse competitiva en el mercado de raíles y planchas. Obtuvo, en promedio, unos beneficios brutos anuales de $£ 98.962$ en la década de los ochenta y de $£ 100.210$ en la de los noventa, invirtieron $£ 250.000$ en instalaciones entre 1904 y 1909 y prosperaron enormemente hasta el término de la Primera Guerra Mundial ${ }^{8}$. Sus trayectorias, sujetas a las mismas limitaciones de mineral de hierro, muestran que otro camino -la estrategia de los dos mercados-, diferente al elegido por Altos Hornos de Vizcaya, era posible.

\section{FUENTES}

Glamorgan Record Office. Cardiff, Gales.

Historisches Archiv Krupp. Villa Hügel-Essen, Alemania.

\section{BIBLIOGRAFÍA}

Allen, Robert C. (1975): «International Competition and the Growth of the British Iron and Steel Industry, 1830-1913». Harvard, MA: Harvard University, Tesis Doctoral.

Alzola y Minondo, Pablo (1896): Memoria relativa al estado de la siderurgia en España, Bilbao: Casa de la Misericordia.

- (1904): Informe relativo al estado de la industria siderúrgica en España y de las reformas requeridas para que alcancen la debida extensión las fábricas de productos derivados y de maquinaria. Bilbao: Casa de Misericordia.

- (1905): El problema industrial. Bilbao: Casa de Misericordia.

Burn, D. L. (1940): Economic History of Steelmaking. Londres: CUP.

Burnham, T. H. y Hoskins, G. O. (1943): Iron and Steel in Britain, 1870-1930. Londres: Allen \& Unwin.

Edwards, J. R. y Baber, C. (1979): «Dowlais Iron Company: Accounting Policies and Procedures for Profit Measurement and Reporting Purposes». Accounting and Business Research 9, pp. 139-151.

Elbaum, Bernard (1986): "The Steel Industry before World War I», en B. Elbaum y W. Lazonick (eds.), The Decline of the British Economy. Oxford: Clarendon Press, pp. 5181.

Houpt, Stefan (1998): «Cambio técnico y localización en la siderurgia española integrada, 1882-1936». Madrid: Universidad Carlos III de Madrid, Tesis Doctoral. .

- (2003): «Competir en los mercados internacionales. Altos Hornos de Vizcaya, 18821936». Revista de Historia Económica XXI (2), pp. 335-372.

${ }^{8}$ Owen (1979, pp. 91, 95 y 100) y Edwards y Baber (1979, p. 150). El promedio de beneficios brutos entre 1856 y 1900 había sido £ 90.347. 
Krupp (1912): Zum 100jährigen Bestehen der Firma Krupp und der Gusstahlfabrik zu Essen. Jena: Gustav Fischer.

McCloskey, D. N. (1973): Economic Maturity and Entrepreneurial Decline. British Iron and Steel, 1870-1913. Cambridge, MA: Harvard University Press.

Monografía AHV (1909). Barcelona: Thomas.

Owen, John A. (1979): The History of the Dowlais Iron Works, 1759-1970. Newport, Starling.

Tolbiday, Steven (1991): «Competition and Maturity in the British Steel Industry, 18701914», en E. Abé y Y. Suzuki (eds.), Changing Patterns in International Rivalry. Some Lessons from the Steel Industry. Tokio: University of Tokyo Press, pp. 20-75.

Webi, Steven B. (1980): «Tariffs, Cartels, Technology and Growth in German Steel Industry, 1879-1914», Journal of Economic History 40 (2), pp. 309-30.

WENGENROTH, Ulrich (1986): Unternehmensstrategien und Technischer Fortschritt. Londres, Vandenhoeck \& Ruprecht. (Traducción al inglés:) (1993): Enterprise and technology: the German and British steel industries, 1865-1895. Cambridge: Cambridge University Press. 